\title{
THE CHANCES OF PROPENSITIES
}

\section{Mauricio Suárez}

\begin{abstract}
This paper argues that if propensities are displayed in objective physical chances then the appropriate representation of these chances is as indexed probability functions. Two alternative formal models, or accounts, for the relation between propensity properties and their chancy or probabilistic manifestations, in terms of conditionals and conditional probability are first reviewed. It is argued that both confront important objections, which are overcome by the account in terms of indexed probabilities. A number of further advantages of the indexed probability account are discussed, which suggest that it is promising as a general theory of objective physical chance. The paper ends with a discussion of the indexical character of the objective chances that are grounded in propensities.
\end{abstract}

1. Introduction

2. Conditional Accounts

\subsection{Conditionals}

2.2. Conditional probability

3. The Indexed Probability Account of Propensities

4. Indexed Chance Functions: History and Comparisons

5. Some Features of the Indexed Probability Account

6. Grounds and Indexes for Propensities

7. Conclusions

Acknowledgements

References 


\section{Introduction}

Propensities, or chancy dispositions, are back on the agenda in both the philosophy of science and metaphysics. They also figure in unsuspected ways in the methodologies of statistical and causal inference (Humphreys [1989]). And they implicitly or explicitly also appear in our ordinary ways of talking and thinking about chancy phenomena. We often say that a particular coin has some propensity to land heads, or tails; that a radium atom has a certain propensity to decay within the hour; that a particular individual has a propensity to smoke, and that smoking has a propensity to cause lung cancer, etc. In all these expressions, $a$ is the propensity property of the object or chance set up, and $b$ is whatever property manifests it - i.e. let us refer to it as the manifestation property. The manifestation of a propensity is essentially probabilistic: A coin is fair, or loaded, and this property of the coin is displayed in each toss in a particular chance of heads or tails. The relevant relation is therefore between a propensity $a$, and the single-case chance over the manifestation property, Prob (b), or Ch (b), that displays the propensity. ${ }^{1}$

This essay provides the outline of a new account of propensities in their relation to chances. Traditionally defenders of propensities have tended to understand "propensity" and "objective probability" as roughly interchangeable terms. On this view, propensities merely provide a model or interpretation for probabilities. Following an influential argument due to Paul Humphreys ([1985], [2004]), a number of philosophers have argued that this is a mistake. An appropriate and fully explanatory philosophical account of physical probability and chancy phenomena requires these two concepts ("propensity" and "chance") to be kept distinct. These two concepts must moreover be each kept distinct from

\footnotetext{
${ }^{1}$ In this essay I do not essentially distinguish 'chances' from the objective probabilities that represent them. Sometimes the distinction is drawn in the literature between chances as metaphysical qualitative entities, and probabilities as formal quantitative ones. I draw the distinction elsewhere, since I reserve the terms 'propensity' for qualitative properties or entities, 'chance' for quantitative ones, and 'objective probability' for the formal representation of the latter.
} 
the further notion of actual (finite) frequency. Propensities explain and ground single case objective chances; chances are represented as probabilities in statistical models; and these models are in turn tested by frequency ratios in sequences of experimental outcomes. I shall here assume the need for a tripartite distinction along these lines, and shall in this paper focus mainly upon the relation between propensities and the single-case chances that display them. I will have very little to say about their links to frequency ratios in experimental statistics.

The focus is therefore squarely on the connection between on the one hand propensities understood as dispositional properties of objects, systems, or chance set ups, ${ }^{2}$ and on the other hand chances, understood as the objective probability distributions that these propensities give rise to every time they are exercised. The relationship between such propensities and the chances that they bring about is thus to be understood on the model of the 'manifestation' relation in the metaphysics of dispositions. However, propensities are inherently probabilistic or chancy dispositions, which means that there must be some irreducibly chancy element that marks them out from ordinary, sure-fire or deterministic, dispositions. ${ }^{3}$ Certainly, chances are peculiar manifestation properties; it is in fact best to describe objective chances as 'displays' over the actual manifestation property. Thus I shall say that propensities - unlike sure-fire or deterministic dispositions - are displayed in chances over the different possible values of some manifestation property (or perhaps more simply its various manifestation properties). Thus a coin's propensity to land heads is displayed in a chance (prob = $1 / 2$ if the coin is fair) of manifesting heads each time it is tossed under normal conditions. The radium atom has a certain half-life $\mathrm{x}$, a propensity which is displayed in a probability or chance $1 / 2$ to manifest decay within a certain period of

\footnotetext{
2 The question of what properties of what systems precisely constitute propensities is beyond the scope of this essay. Addressing it would require both a minimal metaphysics of properties, and empirical research into the particular systems in hand. My hope is to stay neutral in particular on the question as to whether it is just objects, or entire experimental setups that are the recipients of propensities. So I shall refer to both ambiguously as "chance setups". See Hacking ([1965], Ch.2) for further discussion.

${ }^{3}$ In other words, a property is a probabilistic disposition - propensity - if its manifestation is 'chancy': the disposition is displayed in a chance or probability distribution over the values of the manifestation property.
} 
time x. Etc. I shall therefore assume that at least some chances are the displays of underlying propensities; the question is then how to best formally model this 'displaying' relation.

A couple of caveats are in order. Firstly, notice that the discussion is restricted to the relation between the propensities of chancy objects or setups, on the one hand, and their displays in chances or probability distributions, on the other. There is in the vicinity yet another interesting question regarding the relation between these chances and the relative frequencies of outcomes in long run experiments performed on such objects or setups. That is, in addition to the question regarding the relation between the propensities of a coin and its, say, $1 / 2$ chance of landing heads or tails, there is the question regarding the relation of those chances to the relative frequencies of actual outcomes of tossing the coin. This is of course a long-established topic of discussion over which there has been much debate, and it may be supposed that a propensity interpretation of chances must be called in to account for this latter relation. We know from the law of large numbers that in the long run the frequencies will approximate the chances arbitrarily with high probability - and it may well be that this 'higher-level' probability must receive a propensity interpretation. Nevertheless, these are at least prima facie distinct questions, and it is the question of the relation of propensities to chances that will be addressed here. ${ }^{4}$

Secondly, and related, the phrasing of the question above may suggest that the relation between propensities and chances is in fact deterministic. It may suggest that the propensities determine the chances with certainty. Thus the propensities of the coin determine with certainty that the chance of landing heads if tossed is exactly $1 / 2$. There is a long and distinguished tradition that assumes that objective chances are the probabilistic outcomes of deterministic processes acting

\footnotetext{
${ }^{4}$ On matters of the interpretation of the chance or probability functions, I shall adopt an agnostic attitude - or a no-theory theory approach similar to that defended by Elliott Sober (2004). I believe that nothing that I say in this paper is reliant upon any particular interpretation of the chance or probability functions, including Popper's (1959) propensity interpretation of probability. My assumption is that far from interpreting objective probabilities, propensities are in the business of explaining at least some of those probabilities.
} 
over randomly distributed initial conditions - the so-called method of arbitrary functions, originating in Poincaré, Von Kries, etc. ${ }^{5}$ As far as I can tell, everything in this paper is consistent with the assumption, and the account defended may thus be run as a complement to this tradition. Yet, the assumption is not required, and nothing in the account demands underlying determinism. The probabilities or chances may instead simply mediate between propensities and outcomes. The propensities of the coin are thus in some sense responsible for the outcome ('heads' or 'tails') with a certain probability or chance. On this conception the propensities determine the outcomes only up to a certain probability. There is no need to appeal to any underlying determinism, since only one amongst all the possible outcomes happens as a matter of fact. Either conception ('propensities determine chances of outcomes with certainty', or 'propensities determine outcomes with some probability') seems legitimate, and in agreement with our ordinary modal locutions regarding chance, including what we ordinarily seem to mean by 'propensity'. ${ }^{6}$ A good account of chancy propensities should be able to accommodate both.

Section 2 explores two models or accounts for the primary relation of propensities and chances in terms of conditionals, and conditional probabilities respectively. These accounts have in common a critical reliance on some notion of 'conditionality', in the form of either a conditional statement or conditional probability. It is argued that the first account in terms of conditionals confronts important semantic objections, while the second in terms of conditional probability confronts what is known as 'Humphreys' paradox'. Section 3 presents the new account in terms of unconditional indexed probabilities and argues that it overcomes the difficulties that make its competitors untenable. Section 4 compares the account with recent similar views in the literature, and argues against a Humean version. Section 5 reviews in some detail further features and advantages

\footnotetext{
${ }^{5}$ See Von Plato (1983) for an historical account and review.

${ }^{6}$ There is in addition the view that some propensities determine the chances that display them only probabilistically. This seems to pile up probabilities, thus providing a circular definition of the relation of propensities to probabilities, but the circularity is arguably not vicious if the higher-order probability is not itself to be explained as the exercise of any propensity. At any rate, for the sake of simplicity, I leave this option out of the discussion.
} 
of the indexed probability account of propensities and chance phenomena, and applies it in the resolution of a number of different conundrums regarding chance. The final section 6 discusses the ways in which propensities may be said to ground chances according to the indexed probability account, as well as the extent to which the account may be said to provide an indexical - or perspectival - account of chance in general.

\section{Conditional Accounts}

This section critically assesses two accounts of the relation of propensities and chances in terms of conditionals, and conditional probability, respectively. We may refer to (either of) them as 'conditionality' accounts, yet these accounts differ from each other in critical ways, which are very much worth exploring. The first one conceives the relation between propensities and chances as essentially a logical or propositional relation between two statements - specifically the relation between a conditional's antecedent and its consequent. ${ }^{7}$ Probabilities play no essential role in this relation, which can be understood and analyzed irrespective of the calculus of probability. On the second account, by contrast the relation between propensities and chances is itself probabilistic in nature, and the logic of conditional statements plays no essential role. The 'conditionality' aspect in this latter view is rather built into the probability functions themselves, since propensities appear as conditional variables within the chance functions. Hence the nature of the 'conditionality' of chances upon propensities is fundamentally different in these accounts. Nonetheless, there is something that both views share, namely the thought that whatever the nature of the 'conditionality' of chances upon propensities, this 'conditionality' is an intrinsic part of the relation that links

\footnotetext{
7 Throughout the essay I move liberally between a language of propensity and manifestation properties, a language of events ('the obtaining of a certain value of a certain property') or corresponding propositions ('the proposition that a certain property takes a certain value'), and a language of variables representing said properties taking certain values, which in the limit reduce to 0 or 1 depending on whether the property obtains or not.
} 
propensities to their displays, and it must therefore be made formally explicit. I shall argue that 'intrinsic conditionality' ultimately fails, because it generates insurmountable problems for any account of propensities in relation to the chancy displays over their manifestations. Although conditional chances do of course exist and may be well defined, the relation between propensities and the single case chances that display them is not in general provided by any 'conditionality'.

\subsection{Conditionals}

The first account assumes that the relation between a propensity $a$ and its manifestation property $b$ is given by either an indicative or a subjunctive (counterfactual) conditional: 'if a then b', or 'were a to be the case then b'. In the case of propensities, the manifestation property obtains with a certain probability - and is thus displayed in a distribution function over the outcome variables Prob (b) for all outcomes $b$. Thus the simplest model would assume that a propensity property relates to its probabilistic manifestation as follows: If a then Prob (b) (where Prob (b) is the full distribution function over outcomes): 'If the coin is fair then the probability of landing heads is $1 / 2$ and the probability of landing tails is $1 / 2$; 'if the material is made of radium, then the probability that it will decay during the next hour is $0.10^{\prime}$; Etc.

Hugh Mellor ([2005], pp. 53ff.) has proposed a conditional account of the relation between single case chances of events and frequency statistics observed over those very events. Regardless of its merits this account may be considered as a model for the different relation between propensities and chances. Take the wellknown example of radioactive decay. Suppose that radium atoms have a chance $\mathrm{p}_{\mathrm{t}}$ of decaying within t years, where $p_{t}$ is well known to be the inverse of an exponential function of time, such as: $P_{t}=1-e^{-\lambda t}$. Consider then the law:

$\forall x:\left(R x \rightarrow C h\left(D_{t} x\right)=p\right)$, where $R x$ denotes that $x$ is a radium atom, and $C h\left(D_{t} x\right)$ denotes the chance of $x$ decaying in $t$ years. This law seems to appropriately encapsulate the relation between the propensities in radium atoms and their manifested chances of decay. It is typically assumed that there is a reductive basis 
to propensities, such that, say, a radium atom possesses a certain propensity to decay within an hour if it is endowed with a number of fundamental categorical properties that provide a reductive basis for it. Thus in the case of the radium atom, its having a chance $C h\left(D_{t} x\right)$ of decay is identified with its 'having some categorical basis (such as a nuclear structure) for a disposition to produce a hypothetical limiting frequency $\mathrm{p}_{\mathrm{t}}$ of similar R-atoms decaying within $\mathrm{t}$ years' (Mellor [2005], p. 54). The assumption that there is such a categorical basis may be contested. It is particularly inappropriate in the case of quantum mechanics, as applied to the most fundamental constituents of nature, since at that level there is no further level downwards to reduce dispositional properties to. Yet, it turns out to be an unnecessary assumption in this context anyway. The disposition may produce the frequency without any recourse to the categorical basis, and we may simply insist that the law $\forall x\left(R x \rightarrow C h\left(D_{t} x\right)=p_{t}\right)$ holds, where we now assume that ' $\mathrm{R}$ ' denotes merely the possession of the relevant disposition whether or not this is reducible to a categorical basis.

We can thus turn without further ado to the fundamental issue of the form of the law that, on this account, links propensities with their chancy manifestations, regardless of issues of reduction. Mellor does not stipulate the nature of the relation $\rightarrow$ other than it is to be understood as the conditional 'if $\mathrm{R}(\mathrm{x})$ then $C h\left(D_{t} x\right)=p_{t}{ }^{\prime}$. Therefore, this law has exactly the form advertised for the relation of a propensity to its chancy manifestations. In particular, for any chance set up $\mathrm{x}$ it must be the case that 'if $\mathrm{x}$ has a then Prob (b)= $\mathrm{y}$ ' is true, where $a$ is the obtaining of the propensity for $\mathrm{x}$ and Prob $(b)=y$ is the probability over the relevant manifestation property. The only provisos seem to be that i) the probability is understood to define a particular chance value over the manifestation properties, and ii) the manifestation property is suitably timeindexed. Nothing of any fundamental importance depends on these provisos (other than perhaps the assumption that chances are indeed probabilities), and we are still left with the task of understanding the fundamental nature of the conditional that obtains between propensities and their displays. 
The difficulties with the semantics of conditionals are well known (see Edgington [2004]). Roughly there are three accounts: i) Truth-functional; ii) Nontruth functional; and iii) Suppositional accounts. I will here raise two types of difficulty for all of them. Firstly, some general difficulties related to the semantics for conditionals that they presuppose; secondly, specific difficulties with their application to propensities in particular. The main instance of i) is material implication, according to which the conditional 'if a then $b$ ' is false only if ' $a$ ' is true and ' $b$ ' is false, and otherwise it is always true. Most of the problems with this account are related to cases in which the antecedent is false. As regards propensities, in particular, it would be very strange that if an object lacks any propensity then it is true of any of these (absent) propensities that it entails the actual chances, regardless of what these chances are. Any coin that for whatever reason cannot be tossed is such that the coin's non-propensities would entail any chance distribution over heads or tails. This is not normally the way we speak about dispositional properties in general, never mind propensities.

By contrast, on non-truth functional accounts, which apply to subjunctive or counterfactual conditionals, 'if a then $b$ ' may well be false when ' $a$ ' is false. For instance, according to the influential possible world semantics (PWS), the conditional 'if a then $b$ ' is a counterfactual conditional if uttered at any i-world where ' $a$ ' is not the case - and it is then made true or false by facts regarding possible worlds close enough to the i-world. On Stalnaker's (1984) version - which obeys both a uniqueness and a limiting assumption regarding 'close worlds' -, this counterfactual conditional is true at such an i-world if $b$ is true at the a-world (i.e. the possible world where $a$ is the case) closest to this i-world. The virtue of the account is that there is no general truth-functional compositional rule such that all conditionals with false antecedent are true: Some of them are true but others are not, depending on the details of each case. So it is not necessarily the case for any absent propensity that it entails any chance. However, such an account has other problems (see Edgington [2004], pp. 7-8; and Lewis [1973], pp. 77ff).

Stalnaker's limiting assumption entails that there is a limit to the order of closer worlds to any given i-world, so that there exists a certain world W such that 
no other world is closer to the i-world than $\mathrm{W}$. The uniqueness assumption picks out some $\mathrm{W}$ as in fact the closest world to the $\mathrm{i}$-world, and Stalnaker specifies a 'selection function' whose role is to determine W. But why should there be a uniquely specifiable world that is closest to any given i-world? And if so, what may prevent two a-worlds being closest, in one of which $\mathrm{b}$ is true and in the other $\mathrm{b}$ is false? If this cannot be ruled out then it follows that, in general, for any $a$ and $b$, the truth-value of the counterfactual conditional 'had a been the case then $b$ ' is undefined. More particularly, for the conditional account of the relation of propensities and chances, it entails that there is no way to decide, for any propensity statement if a then Prob (b), whether or not in worlds where $a$ is not the case Prob (b) is the chance distribution over the manifestation property variable $b$. In other words, we would never be in a position to determine in advance what chances properly display the propensities of any chance setups.

There does not seem to be much hope for an account of this kind to explain our ordinary locutions and elementary beliefs regarding propensities, if these are understood in the way I have pointed out in their relation to chances. This is because so understood propensity statements are irreducibly modal and essentially tied to their chance manifestations. There is no conceptual room to ascribe propensities with undefined chance displays; and it is irrelevant whether propensities obtain in the actual world or not. We can perfectly imagine a biased coin with a propensity precisely displayed as a chance $1 / 3$ to land heads, even though no real coin in the actual world has precisely that propensity. A counterfactual analysis of such propensity ascriptions, along the lines suggested by Stalnaker's semantics, does not yield any understanding as to what such propensities could possibly consist in. ${ }^{8}$

\footnotetext{
${ }^{8}$ Lewis ([1973], 3.4) provides an alternative account which rejects both the uniqueness and the limiting assumptions and makes no use of any selection function. Although this overcomes some of the problems mentioned in the text and is a more promising approach, it remains problematic. The logic that results does not support conditional excluded middle, so it is not true for any propensity a and manifestation property $b$ that 'if a then prob $(b)=x$ or if a then prob $(b) \neq x^{\prime}$ for any value $x$. This is widely regarded as a problem in general (e.g. Swanson [2011]) and again seems if anything a greater problem for the conditional account of
} 
The third option (iii) for understanding conditionals is the suppositional account suggested in some of Frank Ramsey's writings (Ramsey [1927]). However, this essentially boils down to the idea that a conditional expresses a degree of belief in the proposition $b$ on the supposition of the proposition $a$. This is in other words the second conditionality account in terms of conditional probability, which I turn to next.

\subsection{Conditional probability}

The second conditional account assumes that the relation between propensity properties and manifestation properties is provided by conditional Kolmogorov probability: Prob (b/a). In our example, a fair coin possesses a propensity $(a)$ to land heads $(b)$ with chance $1 / 2$ if and only if Prob $(b / a)=1 / 2$. One major advantage of the account is that it does away with any probabilistic statement in the consequent - it in fact does away with any consequent altogether. The probabilistic manifestation is, as it were, built into the very definition of the propensity instead. This makes the probabilistic relation between $a$ and $b$ conspicuous: it brings to the fore that these two propositions share a probabilistic rather than a propositional or logical structure.

The account has the additional advantage to bring the definition of chancy propensities in line with the ordinary cognitive order: we tend to learn conditional probabilities first, and often possess precise knowledge of conditional chances while lacking any knowledge of the unconditional chances. Thus I may not know whether it will rain tomorrow, but I do know that with high probability my son will visit me if it rains (since we have so agreed beforehand). Or consider the application of our very precise theoretical knowledge of radioactive half-lives to an actual mixed sample of radioactive material. If we do not know the proportions in the mixture with any certainty, we cannot know the actual rate of decay, but this in no way impugns our very precise knowledge of the half-lives for each of the 
radioactive elements that make up the mixture. And as we gain experimental knowledge of the actual rates of decay we can come to estimate rather precisely the composition of the mixture from the conditional chances of decay. It does not seem farfetched to think that at least in some cases and contexts, we may estimate the prevalence of certain propensities in a system from our knowledge of the conditional chances. Thus a system of coins tossed at random may exhibit certain frequencies that we can experimentally determine and then go on to use these to discern the makeup of the system in terms of coins with definite distinct propensities.

However, the conditional probability account also faces very serious problems related to what is known in the literature as "Humphreys' paradox" (Humphreys [1985], [2004]). The problems are by now well-known so I will not describe them in full detail. In particular, we may distinguish two identity theses between propensities and conditional probabilities, which I have referred to in the past as the probability-to-propensity and propensity-to-probability theses (Suárez [2013], [2014]). Both are interestingly false, but only the latter is relevant to the present analysis. The propensity-to-probability thesis states that any propensity may be understood as a conditional probability where the manifestation property is adjudicated a probability conditional on the obtaining of the propensity (more precisely: a conditional probability distribution is laid out over the values of the manifestation property conditional on the propensity - just as required by what I have here called the conditional account of propensities).

Humphreys disproves the propensity-to-probability thesis by means of an ingenious thought experiment (Humphreys [1985], pp. 561ff.) involving photons ejected from a source at time $t_{0}$, impinging upon a half-silver mirror at time $t_{1}$, and transferred and received at some further screen at time $t_{2}$. Humphreys describes the propensities in the thought experiment in detail and denotes them in accordance to the propensity-to-probability thesis as conditional probabilities. He then adds a principle of conditional independence, which is meant to reflect the thought that propensities do not act backwards in time (at least to the extent that causes do not act backwards in time). That is to say that events at times $t_{1}$ or $t_{2}$ 
may not affect the propensities enacted at time $t_{0}$. Humphreys then shows that the propensities in the experiment, expressed as conditional probabilities, together with conditional independence come into conflict with Kolmogorov's axioms, and in particular Bayes' Theorem, or the definition of conditional probability. This means that if chances are to be essentially, or implicitly, defined by Kolmogorov's axioms, the conditional probability account of propensities (the propensity-to-probability half of the identity thesis) must be abandoned. ${ }^{9}$ In other words, no conditional probability account of the relation between propensities and their chancy manifestations can be made to work in general.

\section{The Indexed Probability Account of Propensities}

Humphreys' 'paradox' thus shows implicitly that any attempt to include the propensity attribution event in the domain of the chance function itself is bound to fail. The obvious response is to take the propensity properties out of the domain of the chance or probability function altogether. And it so happens that the obvious response has a lot going for it. On the assumption that propensities are distinct from the chances that display them, the introduction of the propensity attribution events inside the chance function is counter-intuitive anyway. Every propensity is in effect the condition that determines the chances of its manifestation properties. But propensities do not determine their own chances. That is, every propensity in each chance set-up determines a probability distribution over its possible manifestation properties, or outcomes; and this probability precisely represents the chance that displays the propensity. But of course this is not itself the chance of the propensity attribution event. It makes no sense for a propensity to lay out a

\footnotetext{
9 The argument relies on the Kolmogorov axiomatization of classical probability, and some authors have suggested that a possible way to restore the conditional probability account of propensities would be to reject the Kolmogorov axioms, in particular the ratio analysis of conditional probability. There are good independent arguments against ratio anyway (Hájek [2003]). However, the only respectable alternative to Kolmogorov so far is (Renyi [1953]), which turns out to be subject to similar Humphreys-like objections as an account of propensities (Lyons [2014]).
} 
probability over itself - amongst other things because it would be radically incoherent if it were to adjudicate itself a probability zero. ${ }^{10}$

The indexed probability account of propensities is faithful and explicit about the irreflexive nature of the relation between propensities and their displays. If $a$ is the propensity and Prob $(b)$ is the chance that $a$ defines over the possible outcome events $b$, the indexed probability account represents this relation as $\operatorname{Prob}_{\mathrm{a}}(\mathrm{b})$ or $\mathrm{P}_{\mathrm{a}}(\mathrm{b})$, so there can be no doubt that the attribution of the propensity itself lies outside the domain of the chance or probability function. This is a probability function defined by construction over the outcomes of the manifestation property, and explicitly not over the propensity property, which figures in the index. In other words, there is no joint probability of a propensity and its manifestation - the probability of this joint event is not even zero, it is simply ill defined. Furthermore, different propensity properties may define different probability distributions over the same manifestation outcomes. Hence the propensity ( $a$ ) of a fair coin determines a probability $1 / 2$ for heads $(b)$, and similarly for tails. The very same coin may be altered so that it $(c)$ determines a very different probability (chance) for either heads or tails (the coin is loaded). ${ }^{11}$ Therefore in general $\mathrm{P}_{\mathrm{a}}(\mathrm{b}) \neq \mathrm{P}_{\mathrm{c}}(\mathrm{b})$, for the very same object or set-up if endowed with different propensities $a$ and $c$.

The account easily answers the objections that make its competitors untenable. First, the indexed probability account avoids the pitfalls of the conditionals account, in particular it does not follow that any absent propensities entail the chances whatever those chances are. More generally, we are not to understand propensities as antecedents in conditionals with chances in their consequents. The

10 The view that propensities are distinct from the chances that display them has been suggested before - e.g. by Mellor ([1971], Ch. 4). The additional thought that propensities are explanatory of chances has also been echoed in the literature implicitly at least since Peirce ([1878]).

${ }^{11}$ See Keller ([1986]) for a review of the dynamics of coin tossing, and the rather curious fact that it can be modelled by means of two parameters (upwards velocity $v$, and angular velocity $w$ as it is tossed in the air) that are independent of the coin's centre of gravity. Thus one can bias a coin by bending it, but not by altering the symmetry of its mass / weight distribution as is commonly thought. 
relation between a propensity and its display is not in general provided by a conditional statement, and therefore does not rely upon the appropriate semantics for such statements. So no quandaries or disputes regarding the semantics of conditionals may detain us. In particular, we do not need to determine the truth conditions of a counterfactual conditional across any possible worlds.

Second, as for the objections to the conditional probability account, deriving from Humphreys' paradox, the indexed probability account avoids them entirely, since it does not model the relation of propensities and chances in terms of conditional probability. On the contrary, indexes are introduced precisely in order to avoid any conditional probability representation, so there can be no issues regarding the interpretation of inverse conditional probabilities. In particular, note that the fact that Prob $_{a}(b)$ is well defined in no way entails that Prob $b_{b}(a)$ is also well defined. The manifestation property may be included in the domain of the chance or probability function that displays the propensity without in any way requiring that any chance distribution that in turn displays the manifestation property includes in its domain the propensity property that gave rise to it. Suppose that we toss a fair coin and agree to release a radioactive material in the environment only if the coin lands heads. The propensity of the fair coin is displayed in the chance of heads (as $\operatorname{Prob}_{a}(b)=1 / 2$ ), and the event of this particular coin landing heads determines the chance that the radioactive material has of decaying in the environment in the next hour. But the event of landing heads has no propensity to determine whether or not the coin is fair. It cannot determine the chance of its being a fair coin because $a$ is not in the domain of the Prob function so Prob $(\mathrm{a})$ is not defined. There is no reason why the generating conditions or propensities displayed in a chance distribution should themselves receive a chance value under that distribution.

Furthermore, if either a conditional or conditional probability account were in fact applicable to a particular propensity ascription (in line with suggestions to the effect in the previous sections), they would be easily brought under the indexed probability account. More precisely, the propensities they capture would be easily shown to display indexed probabilities after all. The indexed account is 
sufficiently deflationary, in requiring simply that chances be indexed to the propensities that they display, regardless of any logical, causal, or probabilitistic relations that obtain. So the indexed account has then the following significant advantage: It applies always, including whenever no other account applies, but also whenever any other accounts does. It is in other words the only account that applies to all propensities in their relation to their chancy manifestations, independently of any other details or commitments.

As an illustration consider how the reduction would go in the case of a sophisticated conditional account which applied to a particular propensity $\mathrm{R}$ and manifestation property D as: $\forall x\left(R x \rightarrow C h\left(D_{t} x\right)=p_{t}\right)$. Since the conditional takes the form already discussed: if a then Prob (b), we need only turn this into the corresponding indexed probability formulation: Prob $(b)=x$, where $a=R$, and $b=D$. The only loss of generality incurred is the actual conditional statement. Nothing as regards the propensity or the chance that displays it is in any way distinct: The possession of $\mathrm{R}$ is what explains and grounds the chance at time $\mathrm{t}$, and this is best represented on the account here defended as the indexed function: $\operatorname{Prob}_{R}\left(D_{t}\right)=p$. Thus in the example discussed of the radium atom, the solution is to write: $C h_{R}\left(D_{t} x\right)=p$, where we simply truncate the chance function for the value of the chance of decay of radium atoms in particular.

The indexed probability account of propensities does not require an understanding of the relation between propensities and their chancy manifestations as logical consequence, conditional entailment, or any other form of propositional implication (it is rather better conceived of as a kind of explanatory 'grounding'), but it certainly allows such an understanding whenever appropriate. Similarly, for the conditional probability account, whenever applicable: A nonKolmogorov (i.e. non invertible) conditional probability Prob (b / a) can always be transformed into an absolute indexed probability Proba (b) without any loss of generality or information other than the problematic assignment of a probability to the propensity a (or any complex event that results out of the logical combinations of $a$, including its conjunction with another event, such as $a \& b$ ). And 
just as a non-Kolmogorov probability is not automatically invertible, the corresponding indexed probability is also not automatically invertible. So an indexed probability can, within context, also be standing for a conditional nonKolmogorovian probability. In other words, the function $C h_{R}$ (or $\operatorname{Prob}_{a}$ more generally) is itself relative to context: It may sometimes be coding in for a conditional, other times for a non-invertible conditional probability, and yet in other cases it is entirely sui generis.

\section{Indexed Chance Functions: History and Comparisons}

The claim that chance functions are indexed functions is not in itself new, although the thought that they are indexed to propensities certainly is. Thus chance is commonly understood to be relative to time, and the functions that represent chance therefore carry a (sometimes implicit) time index. On the Humean approach defended by David Lewis, for example, chance is a function of the entire state or history of the world up to a certain time, i.e. $\mathrm{Ch}_{H t}(\mathrm{x})$, where $\mathrm{H}_{t}$ is the history of the world $\mathrm{w}$ up to time t. Chance is thus both world and time relative (Lewis [1986], p. 91). Contemporary Humeans who defend a best system account of chance (such as Hoefer [2007]) also tend to conceive of chance as relative to time, since the best system laws that determine the chances are typically dynamical laws parametrized with respect to time. Thus the best system law $\mathrm{L}$ determines or entails the chances for all chancy propositions at every instant in their temporal evolution: $\forall P \forall t: L\left(C h_{t}(P)\right) \rightarrow C h_{t}(P)=x_{t}$. The crucial point is that in accordance with the law the chance of a proposition $\mathrm{P}$ need not be the same at two different times $t, t^{\prime}$, but it will typically differ: $\mathrm{Ch}_{t}(\mathrm{P}) \neq \mathrm{Ch}_{\mathrm{t}^{\prime}}(\mathrm{P})$. Indeed most Humeans believe that the chances of all past events are either zero or one, including those events endowed with non-zero chances at any particular past time relative to the event in question. Thus a fair coin about to be tossed has chance $1 / 2$ of landing heads, but once tossed the chance of it landing heads properly becomes either one or zero at landing (and stays so afterwards). This patently requires implicitly or explicitly indexing every chance function to a point in time in the evolution of the world (or a time-slice thereof). 
The contemporary Humean accounts already understand chance to be an indexed function with respect to both a time $t$ and a world $w$. My proposal is not incompatible with these accounts, but it aims to go further in indexing chance functions not only to their time slices at their worlds, but to whatever propensity they display or manifest as well at that time in that world. Hence I am not proposing to substitute propensity indexes in place of the conventional Humean indexes, but rather to add a further index to those chances that display some underlying propensity. (The proposal does not even require that all chances display propensities, although it is natural to think that all objective chances in fact do. But generality cannot do any harm: If objective chances exist that are not the display of underlying propensities, the index would simply remain empty, or unsaturated, without loss of generality.)

Carl Hoefer ([2007], pp. 564ff.) comes close to the indexed account in his discussion of what he calls stochastic nomological machines (SNMs), which he describes as 'stable, macroscopic chances that supervene on the overall pattern [and] are explicable as regularities guaranteed by the structure of the assumed chance setup' ([2007], p. 564). With the exception of the claim of supervenience upon the Humean mosaic, there is nothing in this statement that a propensity theorist would object to. Hoefer even employs the appropriate terminology in the statement of his stochasticity postulate (SP, a postulate that is also as acceptable to a propensity theorist as to any Humean): 'At many levels of scale (but especially micro-scale), events look "stochastic" or "random", with a certain stable distribution over time; this fact is crucial to the grounding of many objective chances' (Hoefer [2007], p. 563, my italics). While I welcome the emphasis on the grounding of objective chances, the Humean best system analysis of chance defended by Hoefer only describes chances as regularities that supervene on the Humean mosaic (which Hoefer is happy to extend beyond mere spatiotemporal coincidences at the microphysical level to include factual occurring events at 'higher' levels). It does not illuminate the explanatory relation that SNM's hold to 
the chances. ${ }^{12}$ Yet, the relation between the propensities of SNM's and chances is precisely the central focus of the present essay. How does the modal content of the propensities operative in SNM's and the chances that they putatively explain relate? It seems to me that no Humean account of this relation can be provided on pain of circularity - since it would necessarily incur further regularities, whose stability will in turn require explaining by tracing to some SNM or chance setup. The present discussion is an attempt to provide a basic framework for a more promising inquiry into the nature of this grounding relation. ${ }^{13}$

There are other, more recent, claims to the effect that chances are indeed relative to further factors, other than time-slices at particular worlds. Thus (Glynn [2010]) suggests that chances are relative to levels of description. He also comes close to suggesting a formal indexing model to deal with their relative factors. In particular Glynn ([2010], pp. 66ff.) suggests introducing a third subscript in the chance function for any proposition $\mathrm{P}$ as: $C h_{l t w}(P)$. This would index each chance function to a particular time (or time slice) in a possible world at a particular level of description. ${ }^{14}$ By 'levels', Glynn has in mind the emergent ontologies of different

\footnotetext{
12 It is also unclear what role 'nomological machines' actually play in Hoefer's argument. The term was introduced by Nancy Cartwright ([2007]) to describe the arrangement of capacities in an organized whole that thereby yields and explains lawful observable regularities. But as far as I can see nothing in Hoefer's argument depends on chance setups being construed as organized arrays of capacityendowed parts. Rather the appeal to an explanatory structured chance setup is fully consistent with the more general view that chances are grounded in whatever propensities chance setups possess, regardless of their composition or parts. Also capacities in general do not ensue or ground probabilities or chances, while propensities always do. Hence, I stick throughout this article to the more generic and conventional terms 'propensity' and 'chance setup' which seem to me fully in accord with the functioning content in Hoefer's definitions.

${ }^{13}$ It may be thought that Hoefer's claim that propensities are essentially timedirected, and always oriented forwards in time (see e.g. Hoefer, 2007, p. 565; or Hoefer [2011]) cuts against the present indexed probability account. I do not think so. I see no reason why a defender of propensities cannot countenance backwards in time propensities. It seems to me that what is actually hard to countenance is backwards in time chances, but i) it has already been made clear that the indexed account from the start distinguishes chances from the propensities they display, and ii) even the claim that chances are always time directed can be contested (Cusbert [2013]).

${ }^{14}$ Another difference between our proposals is that Glynn seems to be thinking of $l$, $t$, and $w$ as variables in the chance function, since he claims chance is a function of
} 
well-ordered scientific disciplines: the levels of microphysics, macroscopic statistical physics, physical chemistry, molecular and evolutionary biology, geology and geography, psychology, etc. Thus he is able to show that chances at higher levels, e.g. in genetics, are perfectly compatible with an underlying deterministic dynamics at say the microphysical level. As an approach to the conceptual compatibility of chance and determinism this is thoroughly convincing, but one wonders if the levels are always well defined, and if their hierarchy is always uniquely determined. It is not clear, for instance, what hierarchy of levels, if any, operates between geology, on the one hand, and psychology or econometrics on the other. Once the reductionist model to microphysical chances has been given up, there is no obvious hierarchical order among the remaining chances - so an analysis in terms of 'levels' seems limited to only those disciplines that are clearly and uniquely ordered in their ontologies.

My analysis is different since I propose to consider chances in their relation to the propensities in the chance set-ups that they display, whatever level these propensities operate at. This has the advantage over Glynn's proposal to allow for cross-level reductions. For instance, to reduce thermodynamics to statistical mechanics, on my account, would be tantamount to showing how thermodynamic chances or qualities emerge from statistical mechanical dynamical properties; to reduce biology to physics would be tantamount to showing how biological chances are grounded upon physical propensities, etc. One would have to show, in other words, that those chances would appropriately display such underlying propensities at whatever level of description. The proposal to index chances to the propensities of the chance set-up thus cuts across any attempt to relativize them to levels, and delivers us from any problematic assumptions regarding reduction or emergence.

four different variables overall (the fourth one being the proposition that receives the chance in question). My proposal by contrast is resolutely opposed to considering the subscript as a variable in the chance function. It does not suppose that propensities are a function of chances, or vice-versa, if "function" is understood in its precise mathematical sense. 
So while my thesis is similar in spirit to Glynn's, it goes somewhat further and makes 'levels' redundant. This is because propensities already implicitly fix the level of description. I am assuming that propensities are the properties of chance set-ups that ground and explain the chances that display them - and therefore should not be identified with them. (I take it to be a platitude about explanation, whatever its precise form, that nothing can explain that which it is identical with). We don't explain the chances of coins by appealing to quantum mechanical propensities, but by appealing to ordinary properties of macroscopic (classical-mechanical) objects like coins. Similarly, we explain biological chances, e.g. of reproduction or inheritance, by appealing to dispositional biological properties such as fitness variation, heritability, etc., within evolutionary theory (Sober [2013]). And we explain quantum mechanical chances by referring to a theory (quantum mechanics) postulating microscopic entities, properties, and dynamics at the sub-atomic level. Therefore, a chance that is indexed to a propensity is ipso facto also indexed to a specific level of description, often one provided by a particular scientific theory at that level. There is thus no need for any ulterior reference to levels in identifying chances.

\section{Some Features of the Indexed Probability Account}

The indexed account of chancy dispositions, or propensities is deflationary since it does not build any significant theory of the relation between propensities and the chances that display them. It in particular does not assume that propensities cause their displays. Yet, it has some striking formal consequences and features. Perhaps most striking is the asymmetry between indexes and variables in the chance function. A propensity $(a)$ determines a probability or chance distribution over its manifestation property $(b)$ but $b$ does not thereby determine a probability distribution over $a$. This is the sense in which propensities share the asymmetry of causation, as has often been noted. The grounding of chances in propensities is in this respect like causation, yet it does not follow that propensities always or ever cause their manifestations, or that the manifestation relation is in some essential way causal. In other words, the indexed probability 
account reveals clearly that the asymmetry of propensities is sui generis: it is built into its formal representation $a b$ initio. Although sometimes the asymmetry may coincide with a causal one (some propensities may cause their chance manifestations) this need not be the case in general. The coin's propensity is not necessarily a cause of its landing heads in any particular case (and at any rate it is certainly not the total cause). Nor does it seem plausible to suppose that it causes the chance $1 / 2$ in a fair coin. Rather for a toss of the coin to have the single case chance $1 / 2$ the coin must be fair. This propensity is what the single case chance amounts to. Note that this seems in line with dispositions and their manifestations in general, including sure-fire or deterministic dispositions. It would be odd to say that the fragility of a glass caused its breakage, but it seems clear nevertheless that there is an explanatory asymmetry: the breakage cannot explain the fragility but the fragility is certainly often invoked to explain the breakage.

A second important feature worth emphasizing is this: Nothing in the indexed account prevents nested propensities. A propensity a does not fix a chance or probability distribution over itself, only over its manifestation property outcomes $b$, as $\operatorname{Prob}_{a}(b)$; but this does not entail that $a$ cannot in turn be the result of some further propensity $w$. That is, there may be a further propensity $c$ that has $b$ as its manifestation such that $\operatorname{Prob}_{c}(b)=x$. Thus building on our example, a coingenerating machine may have a certain propensity $c$ to produce a fair coin $b$ which in turn has a certain chance $a$ of landing heads when tossed. In other words, the indexed account does not require the manifestation property to be categorical - it too can be a dispositional property. In fact, all properties on this account may be dispositions, or propensities: There could be propensities all the way down.

Thirdly, propensities may combine and interfere with each other, constructively or destructively. Thus suppose that two propensities $a$ and $b$ independently determine chances distributions over the values of some manifestation property $c$ as $\operatorname{Prob}_{a}\left(c_{i}\right)$ and $\operatorname{Prob}_{b}\left(c_{i}\right)$ where $\operatorname{Prob}_{a}\left(c_{i}\right) \neq \operatorname{Prob}_{b}\left(c_{i}\right)$ at least for some $c_{i}$. The joint action of both may then be defined as Prob $b_{a b b}\left(c_{i}\right)$ and it is constructive for $a$ if $\operatorname{Prob}_{a \& b}\left(c_{i}\right) \geq \operatorname{Prob}_{a}\left(c_{i}\right)$ and constructive for $b$ if $\operatorname{Prob}_{a \& b}\left(c_{i}\right)$ $\geq \operatorname{Prob}_{\mathrm{b}}\left(\mathrm{c}_{\mathrm{i}}\right)$; otherwise it is destructive (for either $a$ or $b$ ). For instance, a loaded 
coin with a greater than equal chance of heads constructively interacts with a more intense gravitational field to make it more likely that the coin lands heads when tossed in that environment. And it destructively interacts with the field with respect to tails to make it less likely that it will land tails.

Finally, the indexed probability account has implications for David Lewis' principal principle (PP). The original formulation of this principle (Lewis, [1986], p. 87) appears to be unconditional. It asserts that for any reasonable initial credence function $\mathrm{Cr}$, at any time, the chance $\mathrm{Ch}(\mathrm{A})$ at that time of some proposition A in light of all admissible evidence E should fix the credence: $\operatorname{Cr}(A /(\operatorname{Ch}(A)=x) \& E)=x$. It quickly became clear that PP is nonetheless implicitly conditional. It is first of all conditional on time since both the credence function (Cr) and the chance function ( $\mathrm{Ch}$ ) are understood at a particular time t. In accordance to the indexed probability account, both should properly carry an index. Furthermore, the chance function is to be evaluated at a particular world, since on a Humean account this function is highly sensitive the local matters of fact at that world. And indeed Lewis himself formulates the principle for an indexed chance function as follows (Lewis [1986], p. 97): $C h_{t w}(A)=C r\left(A / H_{t w} T_{w}\right)$, where $C h_{t w}(A)$ is the probability of A that obtains at time $\mathrm{t}$ in world $\mathrm{w} ; \mathrm{H}_{\mathrm{tw}}$ is the history of the world $\mathrm{w}$ up to time $\mathrm{t}$; and $\mathrm{T}_{\mathrm{w}}$ is the theory of chance in world $\mathrm{w}$ (i.e. the entire set of true history-to-chance conditionals in this world). We may refer to this as the original, or old, principal principle ( $\left.\mathrm{PP}_{\text {old }}\right)$, which Lewis abandoned (Lewis [1994]), under pressure from the so-called problem of undermining futures, in favour of one he called the new principal principle $\left(\mathrm{PP}_{\mathrm{new}}\right)$ : $C h_{t w}\left(A / T_{w}\right)=C r\left(A / H_{t w} T_{w}\right)$. The crucial difference is that according to $\mathrm{PP}_{\text {new }}$, the chance of the proposition $A$ is also conditional upon the right theory of chance $T_{w}$ (the true history to chance at world $w$ ). From the perspective of the approach defended in this paper, the move seems unwarranted, and possibly a mistake. Since the theory of chance at world $w$ contains all the propensities at world $w$, it should never appear in the domain of the chance or probability function at all, but should rather appear in the index of the chance function. The appropriate version of the principal principle for propensities $\left(\mathrm{PP}_{\mathrm{prop}}\right)$ would be the following 
statement instead. $\mathrm{PP}_{\text {prop }}: C h_{T_{w}}(A)=C r\left(A / H_{t w} T_{w}\right) . \mathrm{PP}_{\text {prop }}$ states that, in the absence of any inadmissible information about the future, conditional credences at time $t$ track the chances displayed by the propensities that ground them up to time t. ${ }^{15}$

\section{Grounds and Indexes for Propensities}

The relationship between propensities and their chance manifestations is thus not provided by conditionals - it is not so much a logical relation between statements or propositions as an explanatory relation between certain properties of objects or chance setups and their displayed outcomes. Nor is it an essentially causal relation, although it shares many of the features of a causal relation, such as asymmetry, interference, etc. It is not causal in general because it does not seem in general correct that dispositions are the causes of their chancy manifestations.

Thus, consider the archetypical dispositional 'fragility'; we would very reluctantly take this to be a cause of the breaking of any particular fragile object. The cause of the object breaking may be our throwing it down on the floor with sufficient strength, etc. Its fragility is rather more akin to a background condition: it is a fact conducive to, or facilitating of, the actual breaking but it is not its cause.

Dispositions are pre-conditions for the causes that bring about their manifestations, but do not seem to be themselves those causes.

More particularly, the relation is not causal in the following two senses: i) propensities do not cause the chances that display them; ii) chances do not cause the propensities they display. It is not causal in the latter sense because chances do not routinely explain propensities, but the other way round (and we do not suppose effects to explain their causes). And it is not causal in the former sense because even though it is correct to say that propensities are the explanatory

15 This reformulation of the principal principle is incidentally in line with Ismael's ([2008]) convincing argument against including the theory of chance in the domain of the chance function in $\mathrm{PP}_{\text {new. }}$ But while Ismael has to work hard to make the point on behalf of the Humean, the propensity theory of indexed chance defended in this paper has it as a straightforward if not trivial consequence. 
grounds of the chances that display them, they are not so in virtue of any causal relation. What could this explanatory grounding relation then be?

It is instructive at this point to consider the recent literature on 'grounds' (Fine [2000]). A property or set of properties $\{\mathrm{P}\}$ is the grounds for another property $\mathrm{T}$ if and only if $\mathrm{T}$ being the case consists in, or amounts to, $\{\mathrm{P}\}$ being the case. Fine puts the matter in terms of propositions being true (Fine [2000], p. 15), but that marks no significant difference for my purposes here. Thus in Fine's example: 'Its being the case that Britain and Germany were at war in 1940 consists in nothing more than "..." where "..." is a compendious description of the warring activities of various individuals'. Fine argues that 'grounds' so understood are not causal relations or bases; it is not the case that the set $\{\mathrm{P}\}$ is the set of causes of $\mathrm{T}$ : the set of individual actions during the war did not cause the war, it was rather what the war consisted in. Nor is the relation one of logical entailment or analysis: the set $\{\mathrm{P}\}$ is not the analytical sum of all that which makes up the war: those individual actions during the war is what the war consisted in, but they do not provide an analytical definition of the war. For a start the actions are not logically entailed by the war (or vice-versa). Instead the relation is, according to Fine, essentially explanatory: grounds are the 'most metaphysically satisfying' (Fine [2000], p. 22) explanatory link that we may provide for a fact, proposition, property, or condition.

This seems apposite for our purposes and it fits in well with the distinctions that I have been drawing so far. I have been assuming throughout that propensities are distinguished ontologically as the grounds for the chances that display them; and that this is an explanatory and asymmetric relation. The question here, recall, is not about the nature of the frequency data, or even some long run or limiting feature of this frequency. The question pertains to the single case chance that on this view displays the propensity of a fair coin in every single tossing event. What does the chance ascription amount to? I suggest that a particular single case chance of a possible outcome of a manifestation property b: $\operatorname{Prob}_{a}(b)$, consists in the having or possessing of the corresponding propensity by the chance set up that yields it. Thus what it means for a single toss of a fair coin to have the chance $=1 / 2$ 
to land heads is that the coin possesses the corresponding propensity ('fairness'). Yet, this relation is not exactly causal: the propensity $a$ does not cause the probability or chance distribution over this manifestation property, $\operatorname{Prob}_{a}(b)$. Nor does a relation of entailment or conditional, whether indicative or subjunctive, obtain between them: Propensities do not provide an analytical reduction basis for chances. Instead, following the model of 'ground', we may say that the single case chance over the manifestation condition consists in (or amounts to) nothing but possessing the propensity. Moreover, the relation is explanatory, in an extended sense, since the grounds (propensities) explain the chances. Having a particular propensity $(a)$ to land heads $(b)$ if tossed is what the chance $C h_{a}(b)$ consists in. The possession of the propensity is what explains the particular indexed single case chance displayed in that particular case.

This account is in principle applicable only to objective physical chances, i.e. those that physical theories are called in to explain, in line with similar claims for physical dispositions. Thus the fragility of a glass, regarded as a chance propensity, may not cause the long run frequency of the breaking of the glass, but it certainly will figure in its explanation, as a precondition that makes it possible for the glass to break in the proportion in which it does long term. Similarly the propensities that ground and are displayed in chances, will also figure in the explanation of the long run frequencies of the actual manifestation properties.

There remain perhaps a few questions regarding the 'indexed' nature of chancy propensities. I have claimed that the best representation of the relation between propensities and the chances that display them is provided by indexed probabilities. One may wonder to what extent these are akin to 'indexicals' in the philosophy of language. After all, the essential link to the propensity $a$ that grounds it turns the displaying chance $\operatorname{Prob}_{a}(b)$ into a property of the manifestation event relative to the propensity at hand. Let me explain: I toss a fair coin - the chance of heads is $1 / 2$. This chance is a property of the class of events of landing heads, but it is so relative to the particular propensity of the coin that is tossed. If I manipulated the coin by subtly biasing it (e.g. by slightly bending it), I would thereby change the propensities; it would follow that the chance would change, and may no longer be 
$1 / 2$; let us suppose it is now $\operatorname{Prob}_{a}(b)=1 / 3$. This shows that different propensities ground different displaying chances (for the same events).

Yet the converse is also true: the fair coin tossed in a different way (say by a mechanical device that suitably impressed a different force and ensuing trajectory depending on the initial position of the coin) may also be displayed in a chance different from $1 / 2$, let us suppose exactly $\operatorname{Prob}_{a^{\prime}}(b)=1 / 3$, where $a^{\prime}$ is the crucially different propensity of the coin in this particular chance setup. (There is an issue here, which I must put to the side for the moment, as to whether the propensity can be said to be a property of the coin only, or more generally of the coin in the particular chance setup at hand; but note that whatever view we take it generalizes to all properties, including the original propensity $a$, as well as the new $a$ ). In effect, two coins endowed with numerically distinct propensities are nonetheless displayed in the same chance distribution over the same events. This seems to come into conflict with 'grounding', since the chance now appears to be grounded in different propensities. Hence if we wish to maintain the claim, as I do, that the link between propensities and chances is one of explanatory grounding it seems that we are forced to accept that the chances of propensities are 'indexical', in the sense that they are essentially linked to the propensities they display. Thus the equality: $\operatorname{Prob}_{a}(b)=1 / 3=\operatorname{Prob}_{a^{\prime}}(b)$ is only an identity of numerical values, it does not in fact identify the chances themselves. There is therefore a sense in which chances on this view are indexical properties in their relation to propensities. ${ }^{16}$ The statement that 'the chance of such and such a coin to land heads is $1 / 2$ ' is an indexical statement in the sense that it makes an implicit reference to the particular propensity that it displays and to no other. ${ }^{17}$

\section{Conclusions}

\footnotetext{
${ }^{16}$ For helpful discussion of indexicals see, for instance, Braun ([2012]). Note that the only sense in which I argue above that propensities are like indexicals is the relativity of chances to the underlying propensities. I am not arguing for a fuller extent of the analogy. In particular, it does not strike me that the semantics of 'chance' is indexical in the way in which the semantics of 'this' or 'that' is. 17 This may be also characterized as a form of contextualism, similar to the view defended by Handfield and Wilson ([2014]) for Lewis' chances.
} 
I have assumed throughout this essay that at least some physical chances are the displays of underlying propensities. I have argued that the best account of this displaying relation is by means of an indexed probability functions. Chances are relative to the propensities that they display, just as they are relative to times, worlds, levels, or contexts. The account is contrasted with a number of alternatives such as the conditional and conditional probability accounts. Such "conditionality" accounts suffer from some essential difficulties, which are overcome by the indexed probability account (section 3). A number of consequences and features of this account were then outlined (section 4), including the derivation of a novel version of the Lewis' influential principal principle appropriate for propensities. In the final section of the paper, I tentatively suggested a model of the displaying relation as minimal metaphysical grounding which makes sense of the explanatory power of propensities. I also showed that the chances that display propensities have some of the characteristics that we typically ascribe to indexicals - in particular their contextual relativity.

\section{Acknowledgements}

Research towards this paper was funded by the European Commission (MarieCurie project number 329430 (call identifier: FP7-PEOPLE-2012-IEF)) and the Spanish Government (DGCyT project number FFI2014-57064-P), and was conducted within the framework of the ProbPropCond (http://probpropcond.blogs.sas.ac.uk) research project that I directed while employed at the University of London (School of Advanced Study) during the 2013-14 and 2014-15 academic years. I thank the participants at the regular meetings and seminars, particularly Dorothy Edgington, Hugh Mellor, and Luke Glynn, for their comments, as well as the participants in the final workshop entitled "Propensities, Chances and Statistics". Thanks also to audiences at the BSPS 2014 and 2015 conferences, and the EPSA2015 conferences for reactions to related material. 
Mauricio Suárez

Department of Logic and Philosophy of Science,

Faculty of Philosophy,

Complutense University of Madrid,

28040 Madrid, Spain

Email: msuarez@filos.ucm.es 


\section{References}

Braun, D. [2012]: 'Indexicals', Stanford Encyclopedia of Philosophy.

Cusbert, J. [2013]: The Arrow of Chance, PhD Thesis, Australian National University.

Edgington, D. [2004]: 'Indicative Conditionals', Stanford Encyclopedia of Philosophy (revised version 2014).

Feynman, R. [1988]: 'Negative Probability', in F. David Peat and B. Hiley (Eds.)

Quantum Implications: Essays in honour of David Bohm, London: Routledge.

Fine, K. [2000]: 'The Question of Realism', The Philosophers' Imprint, vol. 1, no. 1, June 2001.

Glynn, L. [2010]: 'Deterministic Chance', British Journal for the Philosophy of Science, 61 (1), pp. 51-80.

Hacking, I. [1965]: Logic of Statistical Inference, Cambridge: Cambridge University Press.

Handfield, T. and A. Wilson [2014]: 'Chance and Context', in A. Wilson (ed.), Chance and Temporal Asymmetry, Oxford University Press, pp. 19-44.

Hajek, A. [2003]: 'What Conditional Probabilities Could not Be", Synthese, 137, pp. 273-323.

Hoefer, C. [2007]: 'The Third Way on Objective Probability: A Sceptic's Guide to Objective Chance', Mind, 116, pp. 549-596.

Hoefer, C. [2011]: 'Time and Chance Propensities' in Callender, C. (ed.), Oxford Handbook of Philosophy of Time, Oxford University Press. 
Humphreys, P. [1985]: 'Why Propensities Cannot Be Probabilities', The Philosophical Review, XCIV, 4, pp. 557-570.

Humphreys, P. (1989), The Chances of Explanation, Princeton University Press.

Humphreys, P. (2004), "Some Considerations on Conditional Chances", British Journal for the Philosophy of Science, 55 (4), pp. 667-680.

Ismael, J. [2008]: 'Raid! Dissolving the Big Bad Bug', Nous, 42 (2), pp. 292-307.

Keller, J. B. [1986]: 'The Probability of Heads', The American Mathematical Monthly, 93 (3), pp. 191-197.

Lewis, D. [1973]: Counterfactuals, Oxford: Blackwell.

Lewis, D. [1986]: 'A Subjectivist Guide to Objective Chance', in D. Lewis, Philosophical Papers, Vol II, pp. 83-113.

Lewis, D. [1994]: 'Humean Supervenience Debugged', Mind, 103, pp. 473-490.

Lyons, A. [2014]: 'From Kolmogorov to Popper, to Rényi: There’s no Escaping Humphreys' Paradox (When Generalized)', in A. Wilson, Ed., Chance and Temporal Asymmetry, Oxford University Press, pp. 112-125.

Mellor, H. [1971]: The Matter of Chance, Cambridge: Cambridge University Press.

Mellor, H. [2005]: Probability: A Philosophical Introduction, London: Routledge.

Peirce, C. S. [1878]: 'On the Doctrine of Chances with Later Reflections', in J. Buchler (ed.), Philosophical Writings of Peirce, New York: Dover, $2^{\text {nd }}$ Edition ([1955], Ch. 12, pp. 157ff). 
Popper, K. [1959]: 'The Propensity Interpretation of Probability', British Journal for the Philosophy of Science, 10(37), pp. 25-42.

Sober, E. [2004]: 'Evolutionary Theory and the Reality of Macro Probabilities', PSA 2004 Presidential Address. Also in E. Eells and J. Fetzer, Eds. (2010), The Place of Probability in Science, Boston Studies in the Philosophy of Science, Dordrecht: Springer, pp. 133-162.

Sober, E. [2013]: 'Trait Fitness is not a Propensity, but Fitness Variation is', Studies in History and Philosophy of Biological and Biomedical Sciences, 44 (3), pp. 336-341.

Stalnaker, R. [1984]: Inquiry, Cambridge: Massachusetts: MIT Press.

Suárez, M. [2013]: 'Propensities and Pragmatism', The Journal of Philosophy, 110 (2), pp. 61-92.

Suárez, M. [2014]: 'A Critique of Empiricist Propensity Theories', European Journal for Philosophy of Science 4 (2), pp. 215-231.

Swanson, E. [2011]: 'Conditional Excluded Middle without the Limit Assumption', Philosophy and Phenomenological Research, 95 (2), pp. 301-321.

Rényi, A. [1955]: 'On a New Axiomatic Theory of Probability', Acta Mathematica Academiae Scientiarum Hungaricae, 6, pp. 286-335.

Von Plato, J. (1983), "The Method of Arbitrary Functions", British Journal for the Philosophy of Science, 34 (1), pp. 37-47. 